\section{Percepción en médicos del impacto de la enfermedad cardiovascular en la mortalidad femenina}

\author{
MAURICIO FERNÁNDEZ ${ }^{1,}$ MANUEL PARRA², \\ SEBASTIÁN CALLIGARIS ${ }^{3,4,6}$, VALERIA PARADA ${ }^{1}$, \\ MARÍA JOSÉ OJEDA ${ }^{3,4, a}$, SOFÍA QUIROZ ${ }^{3,4, a}$, CRISTINE CARREÑO ${ }^{3,4, a}$
}

\section{Knowledge among physicians of the main cause of death in women}

Background: There is a misconception that the main cause of death among women is breast cancer, even among physicians, who may neglect cardiovascular preventive measures in this gender. Aim: To assess the knowledge among physicians about the main cause of death among women. Material and methods: A survey was answered by 231 physicians attending a Cardiology and a Gynecology Meeting. Results: Sixty eight percent of respondents indicated that cardiovascular diseases are the main cause of death among women. A higher proportion of specialists than trainees, answered correctly the question ( 72 and $56 \%$ respectively). No gender differences in the answers were recorded. Conclusions: The knowledge about cardiovascular risk in women should be reinforced among physicians.

(Rev Med Chile 2018; 146: 1167-1169)

Key words: Cardiovascular Diseases; Perception; Physicians; Women.
'Departamento de Cardiología, Clínica Alemana. Santiago, Chile. ${ }^{2}$ Departamento Ginecología y Obstetricia, Clínica Alemana. Santiago, Chile.

${ }^{3}$ Centro Medicina Regenerativa. Santiago, Chile

${ }^{4}$ Facultad de Medicina, Clínica Alemana Universidad del Desarrollo. Santiago, Chile. aAlumnas de la carrera de Medicina.

bPhD

Trabajo no recibió financiamiento. Los autores declaran no tener conflictos de interés .

Recibido el 28 de marzo de 2018 aceptado el 24 de mayo de 2018 .

Correspondencia a:

Mauricio Fernández

Departamento de Cardiología,

Clínica Alemana

Vitacura 5159, Vitacura,

Santiago, Chile.

mfernandezr@alemana.cl
L as enfermedades cardiovasculares son la principal causa de mortalidad en los países desarrollados ${ }^{1}$. En Chile, las enfermedades cardiovasculares (ECV) son la principal causa de muerte en mujeres, de acuerdo a resultados publicados por el Instituto Nacional de Estadística en el 2012, manteniéndose la tendencia en el reporte del $2014^{2}$. Esta característica poblacional se observa también en otros países de Latinoamérica como Argentina $^{3}$, Uruguay $^{4}$ y México ${ }^{5}$. Sin embargo, tanto en Chile como en los países mencionados, existe una falsa percepción en la mujer sobre la principal causa de muerte, la que se asocia mayoritariamente con el cáncer de mama ${ }^{3-6}$.

En 2007, en la guía europea de prevención cardiovascular en la práctica clínica, se hace mención al insuficiente reconocimiento de la magnitud de las ECV sobre la mortalidad femenina, incluso en los profesionales de la salud ${ }^{7}$. Sin embargo, en Chile, aún hoy, no existen estudios sobre el tema orientado a los médicos. Teniendo en cuenta el rol esencial de los profesionales de la salud en los programas de prevención cardiovascular ${ }^{8}$, se realizó un estudio exploratorio sobre la percepción de los médicos chilenos (cardiólogos y ginecólogos especialistas o en formación) sobre el impacto de las ECV sobre la mortalidad en las mujeres chilenas.

\section{Material y Métodos}

\section{Universo de estudio}

Médicos asistentes al 3er Congreso de Endocrinología Ginecológica, Reproducción y Andrología en Santiago, Chile del 4 al 6 de mayo de 2017, organizado por la Sociedad Chilena de Endocrinología Ginecológica, y al Curso Anual de Cardiología, en Santiago, Chile, del 29 al 30 de junio de 2017 organizado por la Sociedad Chilena de Cardiología y Cirugía Cardiovascular. 


\section{Instrumento utilizado de evaluación y su modo de empleo}

Se utilizó un cuestionario de dos preguntas de opción múltiple: 1) A su juicio, ¿cuál es la causa más importante de mortalidad femenina? Respuestas: enfermedades oncológicas (EO), cardiovasculares (ECV) o respiratorias (ER); 2) ¿Cuál es la causa más frecuente de muerte en mujeres? Respuestas: cáncer de mama, infarto agudo al miocardio (IAM) o neumonía. Las encuestas fueron realizadas en forma presencial y voluntaria en el momento de la presentación de los asistentes a los Congresos o durante los "Coffe-breaks". Las respuestas fueron analizadas en el grupo general, según nivel de formación y según género. El grupo "médico especialista" está compuesto por cardiólogos y ginecólogos y el grupo "médico especialista en formación" está compuesto por becarios de cardiología, ginecología y medicina interna. En este estudio de percepción no participaron pacientes, ni se recolectó datos personales sensibles, por lo cual no calificó para ser presentado a comité ético científico

\section{Análisis estadístico}

Los datos fueron analizados por medio de tablas de contingencia, con las cuales se estableció la frecuencia de cada variable (respuesta). Para determinar si las diferencias entre las opciones de respuesta son estadísticamente significativas, se aplicó el test exacto de Fischer entre las variables (EO vs ECV y Cáncer de mama vs IAM), omitiendo los resultados de la tercera respuesta, en ambas preguntas, por escasa representación $(<5)$. Se consideró una $\mathrm{p}<0,05$ como significancia estadística. Los datos fueron analizados mediante el programa Prism (GraphPad, Inc.).

\section{Resultados}

Del total de médicos encuestados $(\mathrm{n}=231)$, el $68 \%$ respondió que la principal causa de muerte era la enfermedad cardiovascular y $82 \%$ eligió la opción: infarto agudo de miocardio, como la causa más frecuente de muerte en mujeres (Figura 1). Sin embargo, se observó una diferencia estadísticamente significativa $(\mathrm{p}=0,028)$ en el porcentaje de médicos que eligieron la opción ECV según su grado de formación (médicos especialistas $72 \%$, $\mathrm{n}=179$ vs médicos especialistas en formación $56 \%, \mathrm{n}=52$ ). Los resultados están representados en la Figura 2.

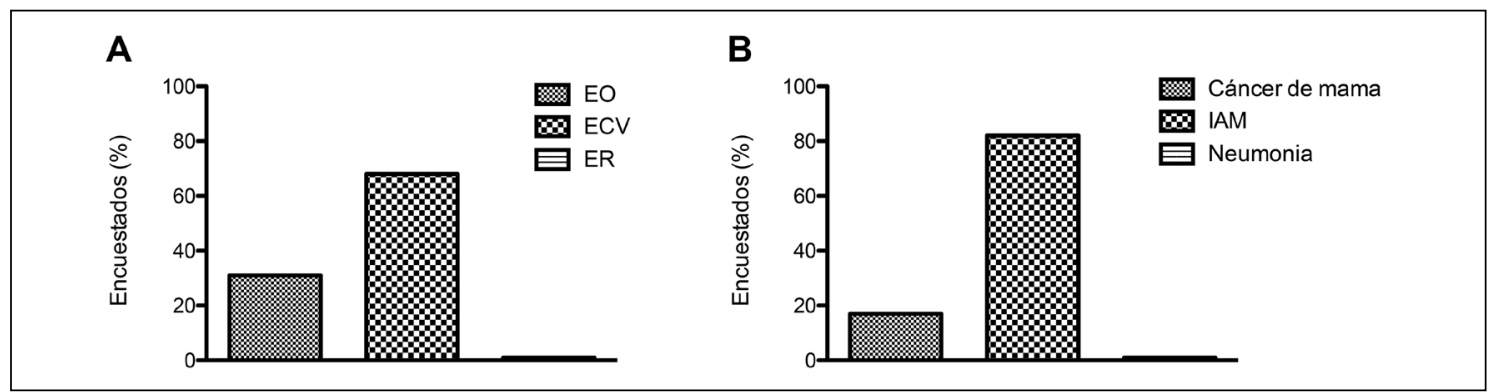

Figura 1. Resultado de la encuesta en médicos. Los resultados de la pregunta 1 y 2 se representan en los gráficos $\mathbf{A}$ y B respectivamente, $n=231$. EO: enfermedades oncológicas, ECV: enfermedades cardiovasculares y ER: enfermedades respiratorias.
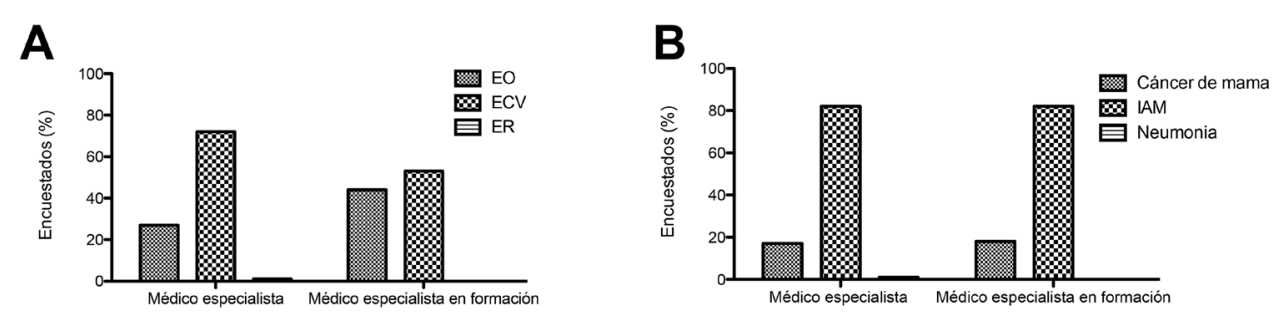

Figura 2. Resultado de la encuesta en médicos según el grado de formación. Los resultados de la pregunta 1 y 2 se representan en los gráficos A y B respectivamente. EO: enfermedades oncológicas, ECV: enfermedades cardiovasculares y ER: enfermedades respiratorias. 
Cuando se analizó las respuestas según el sexo de los encuestados, el porcentaje de médicos que respondieron ECV y IAM fue similar en ambos grupos (Hombres: $75 \%$ y $85 \%, \mathrm{n}=97$ vs Mujeres: $64 \%$ y $79 \%, \mathrm{n}=134$, respectivamente). Por otro lado, se analizaron los datos con relación a la orientación médica y no se encontraron diferencias significativas entre las respuestas de los médicos en la práctica de la cardiología y ginecología (69\% vs 65\% ECV respectivamente, $\mathrm{p}<0,4)$.

\section{Discusión}

En este estudio se muestra por primera vez que el grado de percepción de los médicos sobre la incidencia de las ECV en las causas de muerte en las mujeres chilenas es aún insuficiente (68\%). El año 2017, Bairey Merz y colaborados publicaron los resultados de una encuesta realizada en Norte América a 200 médicos de atención primaria, entre ellos 100 cardiólogos, con la cual se concluyó que solo 39\% de los mismos consideró a las ECVs dentro de los principales problemas de salud en las mujeres, siendo el sobrepeso y la obesidad la preocupación preponderante ${ }^{9}$.

Con respecto a los médicos especialistas en formación, las inconsistencias encontradas en las respuestas entre ambas preguntas sugieren cierta inseguridad en la elección correcta.

Este estudio tiene las siguientes limitaciones: no se analiza el tipo de subespecialidad médica, los años de experiencia laboral, las instituciones donde se ejercen su labor como médicos ni el lugar de residencia. Por lo tanto, es considerado un estudio de investigación exploratorio sobre el tema.

Con este estudio se concluye que además de realizar campañas de concientización a la población en general, en nuestro país serían necesarios cursos o programas de educación o actualización profesional a los médicos, tanto a nivel de pregrado como a nivel de especialidades médicas enfocadas en la salud cardiovascular y la salud femenina como son cardiología y gíneco-obstetricia.

Agradecimientos: A estudiantes de la Univer- sidad del Desarrollo quienes colaboraron en la realizaron de las encuestas. A Sociedad Chilena de Cardiología y Cirugía Cardiovascular (SOCHICAR) y Sociedad Chilena de Endocrinología Ginecológica (SOCHEG).

\section{Referencias}

1. Wold Heart Federation (http://www.world-heart-federation.org) (2018) (Accedido el 29 de junio de 2018).

2. Mortalidad en Chile: 2003 y 2013. Instituto Nacional de Estadística, Chile.

3. Rolandi F, Grinfeld L, Sosa Liprandi MI, Sanvati A. Encuesta de reconocimiento de la enfermdad cardiovascular en la mujer por la mujer. Rev Argent Cardiol 2013; 81: 301-3.

4. Artucio C, Giambruno M, Duro I, Michelis V, Korytnicki D, Barranco D, et al. Enfermedad cardiovascular en la mujer. Cómo la perciben, qué conocen y qué conductas de prevención adoptan las mujeres. Rev Uruguaya Cardiol 2017; 32.

5. Juárez-Herrera L, Castro-Vásquez, M. y Ruíz-Cantero, M. Análisis con perspectiva de género sobre perceopción y prácticas en enfermedad coronaria en mujeres en el norte de México. Salud Pública de México 2016; 58: 428-33.

6. Varleta P, Acevedo M, Casas-Cordero C, Berrios A, Medrano P, Navarrete C. Conciencia de enfermedad cardiovascular en mujeres de la Región Metropolitana a 10 años de la Campaña Mujeres en Rojo (ESTUDIO ESCI). LIV Congreso Chileno de Cardiología y Cirugía Cardiovascular, Santiago, Chile, 2017 (disponible en: http://www.sochicar.cl/index.php/revista-cardiologia.html?layout=edit\&id=3751).

7. Graham I, Atar D, Borch-Johnsen K, et al. European guidelines on cardiovascular disease prevention in clinical practice: executive summary. Atherosclerosis 2007; 194: 1-45.

8. Jaramillo Gómez N. El papel del cardiólogo en un programa de prevención cardiovascular. Revista Colombina de Cardiología 2016; 23: 464-6.

9. Bairey Merz CN, Andersen H, Sprague E, et al. Knowledge, Attitudes, and Beliefs Regarding Cardiovascular Disease in Women: The Women's Heart Alliance. J Am Coll Cardiol 2017; 70: 123-32. 